ORIGINAL ARTICLE

\title{
Comparing intrathecal dexmedetomidine and fentanyl as adjuvants to hyperbaric bupivacaine in elective casarian section, a study.
}

\author{
Sajid Farooq ${ }^{1}$, Muhammad Farhan Ali Rizvi², Syed Muhammad Arslan Yousuf ${ }^{3}$, Rabia Dilshad $^{4}$, Gohar Bashir ${ }^{5}$, Anam Dilshad $^{6}$
}

Article Citation: Farooq S, Rizvi MFA, Yousuf SMA, Dilshad R, Bashir G, Dilshad A. Comparing intrathecal dexmedetomidine and fentanyl as adjuvants to hyperbaric bupivacaine in elective casarian section, a study. Professional Med J 2022; 29(2):218226. https://doi.org/10.29309/TPMJ/2022.29.02.6414

ABSTRACT... Objective: To explore and compare different aspects of fentanyl and DEX when used as adjuvants to hyperbaric bupivacaine in neurexial anesthesia in cases of cesarean sections. Study Design: Randomized Single Blinded study. Setting: Department of Anesthesia, QAMC, Bahawalpur. Period: January, 2019 to December, 2019. Material \& Methods: They were divided in three groups, each group consists of 35 patients having the name of group BN, group BF and group BD. Patients in group BN was given the injection bupivacaine alone, group BF, administered injection bupivacaine along with fentanyl $25 \mathrm{mg}$ and group BD given DEX $10 \mathrm{mg}$ with bupivacaine intrathecally between L4 and L5 intervertebral disc. Scrutiny of onset of sensory block to T5, along with time required to attain Bromage $\mathrm{O}$ scale (motor block). Regression of sensory block (recovery of sensory function) and time required to reach Bromage 3 scale (recovery of motor function) were also recorded. Hemodynamic parameters such as heart rate, systolic and mean arterial pressures along with Ramsay sedation score were also taken into account. Results: Similar demographic profile has been observed in all groups. All three groups differ in terms of onset of sensory and motor block with $p$ value 0.00 when BN was compared with Group BD and BF. Statistically significant results also observed between group BF and group BD with group BD showing shortest time required for initiation of sensory block ( $p$ value .04) and time to reach Bromage $O$ scale ( $p$ value .02). The duration for regression of four sensory segments shows the statistical significance ( $\mathrm{P} 0.000)$ when all three groups were compared, however, no difference found between BF $(175+12.85 \mathrm{~min})$ and BD $(171.88+12.33 \mathrm{~min})$ which showed a P-value of 0.240 . The time required to reach Bromage 3 score was statistically significant between all three groups ( $p$ value 0.00 ) and was also statistically significant between group BF and group BD ( $p$ value 0.00 ) with longest time taken by BD group to reach BROMAGE 3 scale. Both two research groups showed same degree of sedation and comfort for patient. Conclusion: Hyperbaric bupivacaine, in conjunction with dexmedetomidine and fentanyl produced satisfactory results in terms of faster sensory and motor block onset and prolonged time to take in regression of sensory and motor block in comparison to bupivacaine alone. Dexmedetomidine was better among the two adjuncts. Both adjuvants produce same degree of sedation and comfort for patient and relieved apprehension.

Key words: Dexmedetomidine, Fentanyl, Hyperbaric Bupivacaine, Subarachnoid Block.

\section{INTRODUCTION}

Some of the reported advantages of regional techniques include diminished stress response, enhanced, pulmonary and gastrointestinal function, and cost effectiveness. ${ }^{1}$

Whether administered alone into the CSF or in conjunction with a local anesthetic, a number of medications may exert a direct analgesic effect on the spinal cord and nerve roots, and/ or perpetuate the duration of sensory and motor blockade. As such, the co-administration of these agents often allows the estimated dose reduction of local anesthetic, providing the benefit of motor block sparing and faster recovery while still producing the same degree of analgesia.

Central neuraxial opioids have been largely used till date for this purpose. But, now, Intrathecal dexmetomedine (Alpha2 adrenergic agonist) is being extensively evaluated as an alternative to neuraxial Opioids for pain control and has proven
1. MBBS, MCPS, FCPS, Assistant Professor Cardiac Anesthesia, Bahawal Victoria Hospital/QAMC Bahawalpur. 2. MBBS, FCPS, Assistant Professor Cardiac Surgery, Bahawal Victoria Hospital Bahawalpur.

3. MBBS, Medical Officer Anesthesia, BVH, Bahawalpur.

4. MBBS, Resident Medical Officer Gynae/OBS, Bahawal Victoria Hospital Bahawalpur.

5. MBBS, FCPS, Assistant Professor Medical Officer Cardiac Surgery, Cardiac Center Bahawalpur.

6. MBBS, FCPS, Medical Officer Gynecology/OBS, Bahawal Victoria Hospital Bahawalpur.

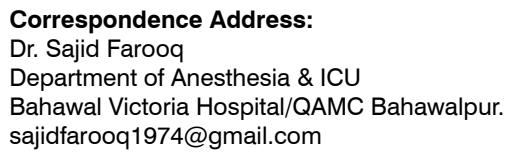

Article received on: 
to be a potent analgesic. That's why; a2-Agonists are gaining popularity as anesthetic adjuvants and analgesics. ${ }^{2}$ The a2 agonist clonidine has been used as an intrathecal supplement to local anesthetics, and this supplement increases anesthetic effects, reduces the amount of local anesthetics ${ }^{3-4}$, and prolongs the extent of sensory and motor block. ${ }^{5-6}$ Dexmedetomidine, a newer a2-Agonists which is 1600:1 more selective for a2 activity compared to a1 in comparison of Clonidine which has a2:a1 activity of $200: 1 .^{7}$

As little as $3 \mu \mathrm{g}$ of dexmedetomidine can prolong motor and sensory block without hemodynamic compromise. ${ }^{8-9}$ This effect is likely elicited by prolonged hyperpolarization of the unmyelinated $C$ fibers (sensory), and to a lesser extent the A fibers (motor function).

Spinal anesthesia is simple and less time consuming procedure to perform with rapid onset and provide better-quality sensorimotor block as well as decreased pain during surgery. Various local anesthetics could be used for spinal blockade; hyperbaric bupivacaine 10 to $15 \mathrm{mg}$ is frequently used to achieve an adequate (T4) block level. Neither patient height nor weight affects block extension ${ }^{10}$, although dosing may require adjustment at extremes of the height spectrum. Adjuncts, such as Fentanyl, Sufentanil, and epinephrine, may be added to amplify the quality of the block. Intrathecal opioids are used for postoperative analgesia in cesarean deliveries.

Fentanyl 5-25 $\mu \mathrm{g}$ in subarachnoid block produced 1-4 hours analgesia and shortened the commencement of sensory and motor block while $0.2 \mathrm{mg}$ morphine yielded up to 20 hours analgesia for cesarean deliveries ${ }^{11}$ while it lengthened the duration. ${ }^{12}$

But opioids are relatively associated with the following side effects: itching, drowsiness, respiratory depression, nausea and vomiting or urinary retention. The appropriate local anesthetic and potential additives must be matched to patients' procedure, regional technique and physician.
Mechanism of action of opioids used for intrathecal injection, described in terms of its affinity to opioid receptors in spinal cord and by reducing the release of substance which is required to transmit pain signals to midbrain but the mechanism of action of much specific in its binding to a2 receptors, dexmedetomidine was not studied exactly. ${ }^{13}$

In Pakistan most commonly used and widely available for subarachnoid block is hyperbaric bupivacaine $7.5 \%$ in dextrose in each $\mathrm{ml}$ of total $2 \mathrm{ml}$ ampoule $(15 \mathrm{mg})$. That is why, we have taken $12 \mathrm{mg}(1.6 \mathrm{ml})$ bupivacaine and added $0.4 \mathrm{ml}$ of adjunctive drugs dose. Injection fentanyl was most widely used as intrathecal in the past as preservative free solution. Now recently introduced injection dexmedetomidine, alpha2 receptor agonist has been used as an adjuvant in subarachnoid block to improve sensory and motor block characteristics and to produce some degree of sedation. By the addition of these adjuvants, the duration and quality of sensory and motor blockade in neuraxial anesthesia were improved and brought more patient satisfaction.

The ambition of the current study was to investigate and compare intrathecal fentanyl and dexmedetomidine as adjuvants to bupivacaine to examine their effects on efficacy, post-operative analgesia, side effects and neonatal conditions in cesarean sections.

\section{MATERIAL \& METHODS}

After obtaining proper written consent and approval from institutional ethical committee, reference no 960/DME/QAMC Bahawalpur, this study was conducted from January, 2019 to December, 2019 in department of anesthesia, QAMC, Bahawalpur. We selected 105 patients. Sample size was estimated by Epi-Info (EpiInfo $^{\mathrm{TM}}$, GA, USA) program. At $79 \%$ power and $95 \%$ confidence interval, the calculated sample was 35 individuals in every group. All patients of age 20 to 30 years who are undergoing first and $2^{\text {nd }}$ caesarian section in subarachnoid block were included in the study. Females were debarred from study who rejected written consent, known to be allergic to any of study drugs, or have 
cerebrovascular, hepatic, cardiopulmonary or renal disease, diabetes mellitus, sepsis or local septic conditions, took prolong analgesic or anticoagulant therapy or have any spinal cord deformities.

Detailed preoperative assessment was done in all patients. General physical systemic examination and airway assessed. All the patients were premedicated with metoclopramide and ranitidine intravenously, half hour before induction of subarachnoid anesthesia.

Group - BN Bupivacaine $12 \mathrm{mg}(1.6 \mathrm{ml})+\mathrm{N} / 10$ (0.4 ml)

Group - BF Bupivacaine $12 \mathrm{mg}(1.6 \mathrm{ml})+$ Fentanyl $15 \mathrm{mg}$

Group - BD Bupivacaine $12 \mathrm{mg}(1.6 \mathrm{ml})$ +DEX $10 \mu \mathrm{g}$

Intervertebral disc space between L4 and L5 was selected for intrathecal injection with a spinal needle of 25 gauges in sitting position. After performing the subarachnoid block, patient is immediately made supine on table. Recording of different parameters were started after successful block. Sensory and motor blockade, were checked and onset time of both the blockade recorded. Routine times monitoring was undertaken as for ASA standard.

Sensory blockade was tested by using pinprick method along the midclavicular line with a blunt 27 gauge needle every 30 seconds till the onset of sensory blockade until the required sensory level of $T_{6}$. Later this sensory level was assessed to be regressed to the level of $T_{10}$ for every 15 minutes. Duration of sensory level of T6 was the time taken from the time of injection till the subject felt sensation at T10. Duration of pain relief was defined as the time from spinal injection to the first request for analgesics (VAS $>5$ ). Injection nalbuphine $10 \mathrm{mg}$ was given $\mathrm{I} / \mathrm{V}$ as rescue analgesia with an adjuvant of injection Provas 1 gram I/V in 15 minutes.

Degree of motor blockade was assessed by modified Bromage score (O weakness, 3 able to move leg or feet). Testing was then conducted every 15 minutes until the complete motor recovery have been achieved (Bromage - $\mathrm{O}$ able to legs against gravity)

Both patient and anesthetist were blinded to the drug used. Sedation was assessed by modified Ramsey sedation scale.

1. Anxious \& restless.

2. Cooperative, oriented, tranquil.

3. Responds to commands only.

4. Brisk response to light glabellar tap.

5. Sluggish response to light.

6. No response.

Hemodynamic monitoring was done every 5 minutes for the first 30 minutes and then every 10 minutes till the shifting of the patient in recovery room using an automated multichannel monitor. Incidence of side effects and time to first rescue analgesic were also noted. Fetal outcome was assessed by Apgar score ( 0 minute and 5 minute). Outcome was taken as good if APGAR score $>=7$ ( 0 minute) and $>=9(5$ minutes $)$.

Statistical analysis was done by using statistical package for social sciences (SPSS version 20). Independent Sample t And ANOVA (F - Test) was used for analysis of continuous variables of two and more groups separately. Categorical data were analyzed using Chi square tests, $\mathrm{P}$ - Value of $<0.05$ was considered significant. The data were expressed as either mean or standard deviation for member and percentages. The demographic data of patients were studied for each of three groups.

\section{RESULTS}

In this present study, we have chosen 105 patients and divided into 35 patients in each of three groups. We have covered different aspects of Apgar score and duration of surgery along with study parameters. Demographic results are proportionate in each of three groups (i.e. age, weight, height, sex, duration of surgery and Apgar score) and showed no statistical significance (Table-I).

In the context of sensory parameters, which 
have been given in Table-II. Considering duration of sensory block reaching at T6 block height, the difference was significant statistically when compared between all three groups each (Chisquare, Friedman test) $[p=0.000]$. Two of the study groups showed obvious difference in getting T6 sensory height in terms of duration as well as statistical significance difference have been observed between Group BF and Group BD groups $[p=0.000]$. Statistically significant results also observed between group $\mathrm{BF}$ and group BD with group BD showing shortest time required for initiation of sensory block ( $p$ value .04). The duration for regression of four sensory segments shows the statistical significance ( $P$ $0.000)$ when all three groups were compared, The time required to reach Bromage 3 score was statistically significant between all three groups ( $p$ value 0.00 ) and was also statistically significant between group BF and group BD ( $p$ value 0.00 ) with longest time taken by $\mathrm{BD}$ group to reach BROMAGE 3 scale. Both two research groups showed same degree of sedation and comfort for patient.

The same statistical difference was observed in terms of regression of sensory block level to T10. See the figure [Figure-1] Groups BD and BF showed better results in respect to control group $\mathrm{BN}$. However, no difference found between BF $(175+12.85 \mathrm{~min})$ and $\mathrm{BD}(171.88+12.33 \mathrm{~min})$ which showed a P-value of 0.240 .

Total time taken to achieve Bromage-0 after administration of successful intrathecal injection was $5.4 \pm 2.19$ in group BF, $4.3 \pm 1.06$ in group BD as compared to control group $\mathrm{BN}$ where this was
$5.8 \pm 1.49$ as shown in Table-II. Here $p$-values are statistical significance ( $p$-values 0.00 ) between all groups comparison and also statistically significant between BF and BD with $p$ value .002 and $B D$ required shortest time to reach Bromage 0 .

Regression of motor blockade to Bromage 3 in intragroup and intergroup have been contemplated highly significant (Table-III). P value of 0.000 was observed in all group comparison and even among groups BD and BF with former resulted in maximum duration of motor blockade.

Now we are explaining hemodynamic parameters which are depicted in table-IV\&V. Giving full attention to this table of hemodynamic observations in terms of heart rate, systolic blood pressure and mean arterial pressure at different time intervals, there have been seen variations but it was not significant statistically by applying Chi-square.

Hypotension was recorded in $11.4 \%$ patients in group $\mathrm{BN}, 17.1 \%$ in group $\mathrm{BF}$ and $14.3 \%$ in group BD. In all patients, hypotension was treated by giving injection phenylephrine $1 \mu \mathrm{g} / \mathrm{kg}$ bolus along with fluid resuscitation. The significance in terms of P-values of sedation scores (Ramsey sedation scale) was high in group BD $34.2 \%$ with a sedation score of 3 and with the same score was observed in $40 \%$ of patients of group BF. No sedation was observed in any of patient of group BN. These incidences of sedation according to Ramsay scale was highly significant showing of P-value of 0.001 .

\begin{tabular}{|l|c|c|c|c|c|}
\hline \multicolumn{1}{|c|}{ Parameters } & Age (Years) & Weight (Kg) & Height (cm) & $\begin{array}{c}\text { Duration of Surgery } \\
\text { [Min] }\end{array}$ & Apgar Score \\
\hline Group Name & $27.4 \pm 4.0$ & $54.73 \pm 6.01$ & $165 \pm 11.3$ & $53.37 \pm 5.92$ & $9 \pm 1$ \\
\hline GBN[n=35] & $26.7 \pm 3.9$ & $53.91 \pm 7.03$ & $166.2 \pm 10.5$ & $52.92 \pm 6.02$ & $8 \pm 2$ \\
\hline GBF[n=35] & $26.0 \pm 4.1$ & $54.21 \pm 6.31$ & $164.7 \pm 10.7$ & $51.89 \pm 7.61$ & $8 \pm 2$ \\
\hline GBD[n=35] & 0.49 & 0.47 & 0.56 & 0.20 & 0.34 \\
\hline p-Value & & & \\
\hline
\end{tabular}

Table-I. 


\section{Group Name}

Onset of Sensory Block In GBN Onset of Sensory Block In GBF

Onset of Sensory Block In GBN Onset of Sensory Block In GBD

Onset of Sensory Block In GBF

Onset of Sensory Block In GBD

Onset of Bromage 0 GBN

Onset of Bromage $0 \mathrm{GBF}$

Onset of Bromage 0 GBN

Onset of Bromage $0 \mathrm{GBD}$

Onset of Bromage $0 \mathrm{GBF}$

Onset of Bromage $0 \mathrm{GBD}$
Mean [min] \pm ST. DEV

$4.35 \pm 1.05$

$3.35 \pm .42$

$4.35 \pm 1.05$

$2.78 \pm 1.41$

$3.35 \pm .42$

$2.76 \pm 1.41$

$5.82 \pm 1.48$

$5.42 \pm 2.18$

$5.82 \pm 1.48$

$4.37 \pm 1.06$

$5.42 \pm 2.18$

$4.37 \pm 1.06$
SIG. (2-tailed)

Chi-Square (F-Test)

.00

.00

0.00

.044

0.05

.001

0.00

Table-II.

\begin{tabular}{|c|c|c|c|}
\hline Group Name & Mean [min] $\pm S T$. DEV & Significance & CHI-square \\
\hline \multirow{2}{*}{$\begin{array}{l}\text { Regression of sensory block GBN } \\
\text { Regression of sensory block GBF }\end{array}$} & $115.2 \pm 37.12$ & \multirow{2}{*}{.00} & \multirow{6}{*}{.00} \\
\hline & $175.8 \pm 12.85$ & & \\
\hline \multirow{2}{*}{$\begin{array}{l}\text { Regression of sensory block GBN } \\
\text { Regression of sensory block GBD }\end{array}$} & $115.2 \pm 37.12$ & \multirow{2}{*}{.00} & \\
\hline & $171.88 \pm 12.33$ & & \\
\hline \multirow{2}{*}{$\begin{array}{l}\text { Regression of sensory block GBF } \\
\text { Regression of sensory block GBD }\end{array}$} & $175.8 \pm 12.85$ & \multirow{2}{*}{.24} & \\
\hline & $171.88 \pm 12.33$ & & \\
\hline \multirow{2}{*}{$\begin{array}{l}\text { Recovery of bromage-3 GBN } \\
\text { Recovery of bromage-3 GBF }\end{array}$} & $132.25 \pm 8.62$ & \multirow{2}{*}{.00} & \multirow{6}{*}{.00} \\
\hline & $144.68 \pm 15.36$ & & \\
\hline \multirow{2}{*}{$\begin{array}{l}\text { Recovery of bromage- } 3 \text { GBN } \\
\text { Recovery of bromage- } 3 \text { GBD }\end{array}$} & $132.25 \pm 8.62$ & \multirow{2}{*}{.00} & \\
\hline & $212.05 \pm 9.75$ & & \\
\hline \multirow{2}{*}{$\begin{array}{l}\text { Recovery of bromage-3 GBF } \\
\text { Recovery of bromage- } 3 \mathrm{GBD}\end{array}$} & $144.68 \pm 15.36$ & \multirow{2}{*}{.00} & \\
\hline & $212.05 \pm 9.75$ & & \\
\hline
\end{tabular}

\begin{tabular}{|l|c|c|c|c|}
\hline \multicolumn{1}{|c|}{ Group-Names } & GBN[n=35] & GBF[n=35] & GBD $[\mathbf{n = 3 5 ]}$ & P-Value \\
\hline Parameters & $80.2 \pm 12.9$ & $81.4 \pm 8.17$ & $87.8 \pm 18.7$ & 0.33 \\
\hline Preop-Dbp & $96.1 \pm 17.1$ & $97.3 \pm 12.8$ & $94.9 \pm 17.4$ & 0.55 \\
\hline Preop-H- Rate & $94.5 \pm 20.9$ & $94.8 \pm 12.3$ & $91.0 \pm 15.1$ & 0.42 \\
\hline Hr At-0 Minutes & $95.9 \pm 18.2$ & $90.9 \pm 13.1$ & $89.0 \pm 16.4$ & 0.29 \\
\hline HR At-5 min & $93.5 \pm 16.6$ & $89.7 \pm 10.3$ & $84.2 \pm 12.8$ & 0.05 \\
\hline HR At-10 min & $90.1 \pm 17.4$ & $91.3 \pm 12.12$ & $81.8 \pm 11.8$ & 0.03 \\
\hline HR At -15 min & $91.9 \pm 18.6$ & $87.2 \pm 12.16$ & $82.5 \pm 12.6$ & 0.20 \\
\hline HR At-30 min & $93.1 \pm 17.3$ & $89.1 \pm 10.89$ & $85.4 \pm 13.2$ & 0.08 \\
\hline HR At-45 min & $77.9 \pm 4.7$ & $76.3 \pm 4.05$ & $74.9 \pm 4.19$ & 0.21 \\
\hline HR At-60 min & $74.5 \pm 20.9$ & $73.7 \pm 11.04$ & $72.0 \pm 15.1$ & 0.09 \\
\hline HR At-80 min & $77.2 \pm 4.9$ & $73.8 \pm 2.69$ & $74.8 \pm 3.36$ & 0.03 \\
\hline HR At-100 min & & Table-IV. & & \\
\hline
\end{tabular}




\begin{tabular}{|c|c|c|c|c|}
\hline $\begin{array}{l}\text { Group-Names } \\
\text { Parameters }\end{array}$ & GBN & GBF & GBD & P-Value \\
\hline PREOP-SBP & $130.5 \pm 14.98$ & $127.9 \pm 11.55$ & $133.7 \pm 13.88$ & 0.26 \\
\hline SBP-5 min & $91.4 \pm 28.27$ & $93.3 \pm 19.99$ & $99.4 \pm 27.64$ & 0.51 \\
\hline SBP-10 min & $104.7 \pm 2.69$ & $106.5 \pm 2.29$ & $107.4 \pm 2.95$ & 0.01 \\
\hline SBP-15 min & $93.6 \pm 15.03$ & $97.2 \pm 14.74$ & $109.4 \pm 33.15$ & 0.19 \\
\hline SBP-30 min & $111.2 \pm 3.76$ & $114.5 \pm 5.07$ & $114.2 \pm 4.25$ & 0.06 \\
\hline SBP-45 min & $114.9 \pm 4.10$ & $114.5 \pm 5.07$ & $113.05 \pm 4.41$ & 0.24 \\
\hline SBP-60 min & $113.7 \pm 4.55$ & $114.6 \pm 4.19$ & $115.4 \pm 3.09$ & 0.08 \\
\hline SBP-80 min & $115.5 \pm 4.46$ & $116.0 \pm 4.15$ & $114.2 \pm 4.69$ & 0.32 \\
\hline SBP-100 min & $116.6 \pm 4.54$ & $115.5 \pm 4.19$ & $114.5 \pm 3.94$ & 0.14 \\
\hline \multicolumn{5}{|c|}{ Mean Arterial Pressures } \\
\hline MBP/-5 min & $64.1 \pm 3.54$ & $65.1 \pm 3.16$ & $63.5 \pm 3.44$ & 0,06 \\
\hline MBP-10 min & $59.5 \pm 2.26$ & $61.17 \pm 3.50$ & $61.4 \pm 3.19$ & 0.04 \\
\hline MBP-15 min & $65.1 \pm 2.53$ & $63.14 \pm 3.50$ & $62.0 \pm 3.49$ & 0.67 \\
\hline MBP-30 min & $64.6 \pm 2.48$ & $64.28 \pm 3.46$ & $65.5 \pm 2.95$ & 0.13 \\
\hline MBP-45 min & $62.1 \pm 2.59$ & $64.13 \pm 3.48$ & $63.2 \pm 3.08$ & 0.08 \\
\hline MBP-60 min & $64.5 \pm 2.59$ & $63.3 \pm 3.11$ & $61.9 \pm 3.08$ & 0.16 \\
\hline MBP-80 min & $60.5 \pm 2.81$ & $64.3 \pm 3.14$ & $62.1 \pm 3.08$ & 0.22 \\
\hline MBP-100 min & $63.8 \pm 3.44$ & $65.14 \pm 2.61$ & $65.2 \pm 2.99$ & 0.11 \\
\hline
\end{tabular}

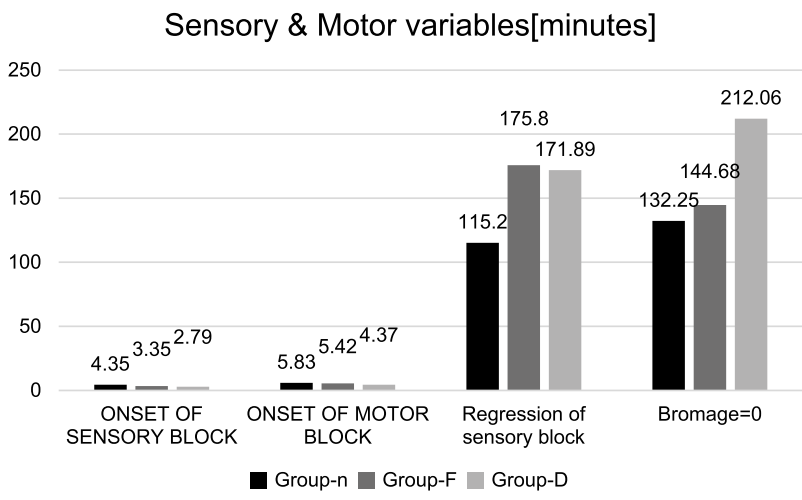

Figure-1.

\section{DISCUSSION}

In our research, we observed statistically significant results in view of duration of sensory and motor block between all the three groups except onset of motor blockade, except between onset of motor blockade between control group $\mathrm{N}$ and study group $F(P$ 0.610). In the same way, the results for regression of sensory height between all three groups found statistically significant except no any significant difference in research groups BF and BD. Same results are shown in all groups in terms of regression of motor blockade.

In a research by Sushruth MR. et $\mathrm{al}^{13}$, the onset of sensory block was significant between DEX

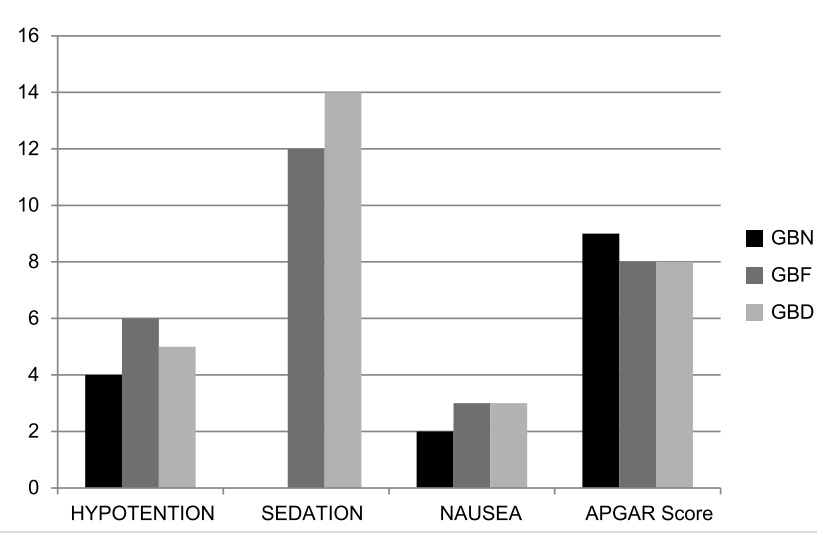

Figure-2. Untoward effects.

group and control group as well four segments regression of sensory block was extended in DEX group (PS 0.001). These results are same as shown in this study, between study and control group. Time to reach Bromage zero and regression of motor blocks to Bromage 3 were significant statistically ( $P$ 0.001). These all results between control and DEX group were equivalent with our study. Hemodynamic variables observed the same pattern as seen in the present study in case of heart rate, SBP, MBP and DBP (Table).

In a study by Rajin Gupta et $\mathrm{al}^{14}$, the sensory and motor blockade onset and regression characteristics were following the same 
characteristics as given in this study. Hemodynamic characteristics also followed the same pattern as shown in current study.

In one classic study by Al-Ghanem et al..$^{15}$ viewed that DEX group achieved early sensory and motor blockade than group fentanyl, thus, coinciding our study, whereas mean time required for reversion of sensory block was $274+73$ min in DEX group and 179+47 min in fentanyl group (P 0.001) also collate with our study. Motor blockade reversion was also statistically significant (P0.001) with DEX group took more time to revert to Bromage 3 scale which again correlate with present study.

In another original article by Venkanna Pocham ${ }^{16}$, onset and duration of sensory blockade were comparable and it is more significant in dexmedetomidine group as compared to fentanyl group $F$. The same results in terms of duration of sensory and motor blockade and revert back of sensory level to 5 segment regression and Bromage $\mathrm{O}$ were shown as observed in the article by and same outcome have been seen in terms of hemodynamic and modified Ramsey sedation grade.

Mahmoud $\mathrm{M}$ Amer et $\mathrm{al}^{17}$ concluded in his study that mean arterial pressure and heart rate during intraoperative period showed no statistical difference between all three study groups. Fentanyl and dexmedetomidine administered intrathecally prolonged the sensory and motor blockade with a time to reach highest sensory blockade were 4 minutes in DEX group and 4 minutes in fentanyl group as well as regression times were $392+43.1$ in DEX group and $324+$ 73.0 in fentanyl group. Same observations were seen in case of Bromage scale in his study, in our study the results are similar with above mentioned study except regression of sensory blockade at T10.

If we see some other studies ${ }^{18,19}$ where Bhure AR et al. concluded the same significant outcome in fast onset of motor and sensory blockade with dexmedetomidine and prolong duration of both sensory and Bromage $\mathrm{O}$ along with stable hemodynamics. In Bhures study, he compared only dexmedetomidine in two different doses with control (Bupivacaine and saline only).

Kamali $A^{20}$, in Iran conducted a study with dexmedetomidine and fentanyl to see the effects as an adjuvant to lignocaine in post cesarean section analgesia and he reached the conclusion that the duration of analgesia prolonged significantly.

Conclusion drawn by Binod Gautam ${ }^{21}$ et al was comparable with our research where dexmedetomidine was superior than fentanyl group in duration of analgesia.

Rajni Gupta ${ }^{14}$ and coworkers carried out same like us with $12.5 \mathrm{mg}$ bupivacaine $+25 \mathrm{mg}$ fentanyl and dexmedetomidine 5 microgram in 12.5 mg hyperbaric bupivacaine and they reached on conclusion that longer time was taken by dexmedetomidine (P-0.001) of sensory block as compared to fentanyl. In his study, duration of sensory reversion to $S 1$ was $476+23$ minutes in group DEX and $187+12$ minutes in group fentanyl $(F)$. Motor regression time to Bromage scale zero noted $421+21$ minutes and $149+18$ minutes in fentanyl group (0.001).

These all above given results are correlating with our study where onset of T5 sensory time and Bromage3 were prolonged significantly as well as regression of motor blockade to get power of Bromage3 was $212.05+9.75$ in group BD and $144.68+15.36$ minute in fentanyl groups. Shujun et al, in a meta-analysis also showed superiority of DEX over Fentanyl in prolongation of sensory and motor block and pain free period post operatively. ${ }^{22}$

Therefore it has been seen from all above mentioned discussion that adding adjuvants in the form of dexmedetomidine and fentanyl achieved fastest onset of sensory blockade and prolonged duration of sensory and motor blockade along with greater hemodynamic stability and some degree of sedation. Ramsey sedation scale brought patient comfortability with these adjuvants with hyperbaric bupivacaine and a very less side effects i.e. hypotension and 
bradycardia noted and treated simultaneously.

\section{CONCLUSION}

Hyperbaric bupivacaine, in conjunction with dexmedetomidine and fentanyl produced better results as compared to alone. Dexmedetomidine was found best in sense of fastest sensory and motor block onset and prolonged time to take in regression of sensory and motor block. These two adjuvants produce same degree of sedation, comfortable for patient and relieved apprehension.

Copyright $\odot 21$ May, 2021.

\section{REFERENCES}

1. Lee KH, Lee SJ, Park JH, Kim SH, Lee H, Oh DS et al, Analgesia for spinal anesthesia positioning in elderly patients with proximal femoral fractures: Dexmedetomidine-ketamine versus dexmedetomidine-fentanyl. Medicine. 2020; 99(20):e20001.

2. Liu X, Zhang X, Wang X, Wang J, Wang H. Comparative evaluation of intrathecal bupivacaine alone and bupivacaine combined with dexmedetomidine in cesarean section using spinal anesthesia: A metaanalysis. Journal of International Medical Research. 2019; 47(7):2785-99. doi: 10.1177/0300060518797000.

3. Bajwa BS, Singh AP, Rekhi AK. Comparison of intrathecal clonidine and fentanyl in hyperbaric bupivacaine for spinal anesthesia and postoperative analgesia in patients undergoing lower abdominal surgeries. Saudi journal of anaesthesia. 2017; 11(1):37.

4. Sharan R, Verma R, Dhawan A, Kumar J. Comparison of clonidine and fentanyl as adjuvant to ropivacaine in spinal anesthesia in lower abdominal surgeries. Anesthesia, essays and researches. 2016; 10(3):526.

5. Boussofara $M$, Carlès $M$, Raucoules-Aimé $M$, Sellam MR, Horn JL. Effects of intrathecal midazolam on postoperative analgesia when added to a bupivacaine-clonidine mixture. Regional Anesthesia \& Pain Medicine. 2006; 31(6):501-5.

6. Carollo DS, Nossaman BD, Ramadhyani U. Dexmedetomidine: A review of clinical applications. Current Opinion in Anesthesiology. 2008; 21 (4):457-61. doi: 10.1097/ACO.0b013e328305e3ef.
7. Shaikh SI, Revur LR, Mallappa M. Comparison of epidural clonidine and dexmedetomidine for perioperative analgesia in combined spinal epidural anesthesia with intrathecal levobupivacaine: A randomized controlled double-blind study. Anesthesia, essays and researches. 2017; 11(2):503.

8. Bi YH, Cui XG, Zhang RQ, Song CY, Zhang YZ. Low dose of dexmedetomidine as an adjuvant to bupivacaine in cesarean surgery provides better intraoperative somato-visceral sensory block characteristics and postoperative analgesia. Oncotarget. 2017; 8(38):63587-95.

9. Liu L, Qian J, Shen B, Xiao F, Shen H. Intrathecal dexmedetomidine can decrease the $95 \%$ effective dose of bupivacaine in spinal anesthesia for cesarean section: A prospective, double-blinded, randomized study. Medicine. 2019; 98(9):e14666.

10. Karaman S, Günüsen I, Uyar M, Biricik E, Fırat V. The effects of morphine and fentanyl alone or in combination added to intrathecal bupivacaine in spinal anesthesia for cesarean section. Agri. 2011; 23(2):57-63.

11. Shah OM, Bhat KM. Comparison of the efficacy and safety of morphine and fentanyl as adjuvants to bupivacaine in providing operative Anesthesia and postoperative analgesia in Subumblical surgeries using combined spinal epidural technique. Anesthesia, essays and researches. 2017; 11(4):91320.

12. El Aish KA, Tafish $R$, Zourob $H$. Morphine versus fentanyl for spinal post-caesarean analgesia: A randomised controlled trial. The Lancet. 2018; 391:S20. doi: 10.1016/S0140-6736(18)30386-6

13. Sushruth MR, Rao DG. Effect of adding intrathecal dexmedetomidine as an adjuvant to hyperbaric bupivacaine for elective cesarean section. Anaesthesia, Pain \& Intensive Care. 2019; 22(3):348-54.

14. Gupta R, Verma R Bogra J,Kohli M and Kushwala JK. A comparative study of intrathecal dexmedetomidine and fentanyl as adjuvants to Bupivacaine. J Anaesthesiol Clin Pharmacol. 2011; 27(3):339-43.

15. Al-Ghanem SM, Massad IM, Al-Mustafa MM, Al-Zaben KR, Qudaisat IY, Qatawneh AM, et al. Effect of adding dexmedetomidine versus fentanyl to intrathecal bupivacaine on spinal block characteristics in gynecological procedures: A double blind controlled study. Am J Appl Sci 2009; 6:882-87.

16. Pocham V, Naik LG. A Prospective Randomized double blind study comparing intrathecal dexmedetomidine and fentanyl as adjuvants to bupivacaine in infra Umbilical surgeries. Int J Sci stud 2018:6(7):1-14. 
17. Mahmoud M Amer, Doaa A Rashwan, Maha A Shaker. Comparative study of two doses of intrathecal dexmedetomidine versus fentanyl as adjuvant to hyperbaric bupivacaine spinal anesthesia. Medicine Science 2015;4(3):2450-64: doi: 10.5455/ medscience.2015.04.8240

18. Bhure AR. Ketaki S. Marodkar*, Sumita Bhargava Pramanik. A comparative study of two doses of intrathecal dexmedetomidine $10 \mathrm{mcg}$ and $15 \mathrm{mcg}$ as adjuvants to $0.5 \%$ hyperbaric bupivacaine for abdominal hysterectomy: A randomized, prospective, double blind study. Int J Basic Clin Pharmacol. 2016 Oct; 5(5):2215-21.

19. Mahendru V, Tewari A, Katyal S. A comparison of intrathecal dexmedetomidine, clonidine, and fentanyl as adjuvants to hyperbaric bupivacaine for lower limb surgery: A double blind controlled study. J Anaesthesiol Clin Pharmacol. 2013; 29:496-502.
20. Kamali A, Azadfar R, Pazuki S, Shokrpour M. Comparison of dexmedetomidine and fentanyl as an adjuvant to lidocaine $5 \%$ for spinal anesthesia in women candidate for elective caesarean. Open Access Maced J Med Sci. 2018; 6(10):1862-67.

21. Binod Gautam, Sushila Tabdar, Ujma Shrestha. Comparison of fentanyl and dexmedetomidine as intrathecal adjuvants to spinal anaesthesia for abdominal hysterectomy; J Nepal Med Assoc 2018; 56(213):848-55.

22. ShuJun Sun. JiaMei wang. NaRen Bao, Ying Chen, Jun wang; Comparison of dexmedetomidine and fentanyl as local anesthetic adjuvants in spinal anesthesia: A systematic review and meta-analysis of randomized controlled trials; Drug Design, Development and Therapy 2017; 11:3413-24.

\begin{tabular}{|c|l|l|l|}
\hline \multicolumn{3}{|c|}{ AUTHORSHIP AND CONTRIBUTION DECLARATION } \\
\hline No. & \multicolumn{1}{|c|}{ Author(s) Full Name } & \multicolumn{1}{|c|}{ Contribution to the paper } & Author(s) Signature \\
\hline 1 & Sajid Farooq & Main author \\
\hline 2 & M. Farhan Ali Rizvi & Contributor \\
\hline 3 & Syed M. Arslan Yousuf & Contributor \\
\hline 4 & Rabia Dilshad & Contributor \\
\hline 5 & Gohar Bashir & Contributor \\
\hline 6 & Anam Dilshad & Contributor \\
\hline
\end{tabular}

